\title{
Large-scale fabrication of achiral plasmonic metamaterials with giant chiroptical response
}

\author{
Morten Slyngborg, Yao-Chung Tsao and Peter Fojan*
}

\author{
Full Research Paper \\ Address: \\ Department of Physics and Nanotechnology, Aalborg University, \\ Skjernvej 4A, 9220 Aalborg East, Denmark \\ Email: \\ Peter Fojan ${ }^{*}$ - fp@nano.aau.dk \\ * Corresponding author \\ Keywords: \\ biomolecule sensing; extrinsic chiral metamaterials; scalable \\ fabrication
}

Beilstein J. Nanotechnol. 2016, 7, 914-925. doi:10.3762/bjnano.7.83

Received: 01 April 2016

Accepted: 09 June 2016

Published: 24 June 2016

Associate Editor: A. J. Meixner

(ㄷ) 2016 Slyngborg et al.; licensee Beilstein-Institut. License and terms: see end of document.

\begin{abstract}
A variety of extrinsic chiral metamaterials were fabricated by a combination of self-ordering anodic oxidation of aluminum foil, nanoimprint lithography and glancing angle deposition. All of these techniques are scalable and pose a significant improvement to standard metamaterial fabrication techniques. Different interpore distances and glancing angle depositions enable the plasmonic resonance wavelength to be tunable in the range from UVA to IR. These extrinsic chiral metamaterials only exhibit significant chiroptical response at non-normal angles of incidence. This intrinsic property enables the probing of both enantoimeric structures on the same sample, by inverting the tilt of the sample relative to the normal angle. In biosensor applications this allows for more precise, cheap and commercialized devices. As a proof of concept two different molecules were used to probe the sensitivity of the metamaterials. These proved the applicability to sense proteins through non-specific adsorption on the metamaterial surface or through functionalized surfaces to increase the sensing sensitivity. Besides increasing the sensing sensitivity, these metamaterials may also be commercialized and find applications in surface-enhanced IR spectroscopy, terahertz generation and terahertz circular dichroism spectroscopy.
\end{abstract}

\section{Introduction}

In recent years metamaterials have attracted a tremendous amount of attention owing to their unique properties enabling the fabrication and design of devices hitherto impossible. These properties have found implementations in various fields such as optics [1], improved photovoltaic devices [2], electronics [3], surface-enhanced infrared spectroscopy [4], Raman spectroscopy [5] and biosensors [6].
Planar chiral metamaterials (PCMs) have also attracted attention because of their negative refractive index [7,8] and optical activity [9] such as circular dichroism (CD) [10]. Among other things, this makes them useful for the production of sensing devices for organic molecules and biomolecules [10]. Recently, the phenomena of the long proposed extrinsic chirality dating back to 1945 [11] have been observed experimentally with ma- 
terials that are achiral [12]. These extrinsic chiral metamaterials (ECMs) demonstrate CD responses that are orders of magnitudes larger than their PCM counterpart [12]. ECMs typically consist of achiral subwavelength hole arrays where the chiroptical response originates from a large area excitation of surface plasmon polariton (SPP) waves. Compared to the localized surface plasmon resonance from PCMs, SPP waves from ECMs are extremely sensitive to the angle of incidence and less sensitive to structural imperfections [13]. Furthermore, ECMs are defined by having a zero response angle, which is the angle where the ECMs exhibit mirror symmetry and hence do not yield a CD response. Though some PCMs show promise as they also yield huge CD responses through FANO resonance [14], the greatest advantage of ECMs is that sensing of biomolecules can be performed with only one sample in one experiment by inverting the tilt of the sample, whereas PCMs requires one samples of both enatiomeric structures and independent experiments with both samples.

To date, only very few different ECMs other than hole arrays $[13,15,16]$ and the original suggested U-shaped and split ring structures [12], have been investigated. These have been thoroughly studied [17-20] and other structures including theoretical suggestions are limited to plasma sphere arrays [21], gold dot arrays [22], gold square arrays [23], metal disk arrays [24], two layer hole arrays [25], polystyrene sphere templates for gold deposition [26] and structures formed by carbon nanotubes [27].

However, in order to be able to apply these metamaterials in sensing devices of organic molecules and biomolecules a reliable large-area fabrication method is required. State-of-the-art fabrication techniques are based on electron beam lithography or focused ion beam milling, which both are expensive and time consuming methods. Large-scale fabrication of PCMs have been attempted to some degree applying different approaches such as glancing angle deposition [28], scaffold ornamentation [29,30], individual chiral nanoparticles [31], preassembled nanoparticles [32-34] and a variety of colloidal nanolithography techniques [35-37]. Compared to the above mentioned PCMs, the experimentally proven ECMs only comprise structures from polystyrene sphere templates for gold deposition [26], structures formed by carbon nanotubes [27] and larger U-shaped structures by nanoimprint lithography (NIL) [38] which have been scalably fabricated.

In the present work we present a novel route towards the largescale fabrication of ECMs and metamaterials in general. These structures have never been reported before and add to the scarce amount of experimentally investigated ECMs. Our fabrication approach is based on a two-step thermal NIL process with subsequent glancing angle metal deposition. The master mold for the NIL was fabricated by anodic oxidation of an aluminum (Al) substrate, which has been demonstrated previously $[39,40]$. During this process a disordered honeycomb structure is formed in the substrate. By controlling different parameters the interpore distance has been varied. ECMs with different interpore distance were investigated with CD spectroscopy and scanning electron microscopy (SEM). Furthermore, by altering the interpore distance and the glancing angle for metal deposition it was possible to tune the obtained CD signals from UV all the way to IR wavelengths. As a proof of concept, the resonance shifts of SPPs were studied upon interactions between a protein or a chiral organic molecule and the ECM surface.

\section{Results and Discussion}

The ECM fabrication process is illustrated schematically in Figure 1. The original mold was fabricated by a self-ordering anodic oxidation of $\mathrm{Al}$ foils as described in a previous study [39]. In this process pores are produced in the Al surface with a honeycomb structure. However, the pattern is not perfect and several types of defects are present, which are inevitably transferred to the final ECMs. By applying different acids and voltages in the anodic oxidation process, original molds with three different interpore distances (300, 430 and $600 \mathrm{~nm}$ ) have been prepared as previously described [40]. These served as master molds in the following NIL process (Figure 2a).

The first imprints were cast in TOPAS polymer substrates. After de-molding the TOPAS structures were sputter-coated with a thin Al film and subsequently an anti-stick monolayer was applied to produce negative molds (Figure $2 \mathrm{~b}$ ). The negative molds were then cast in PMMA polymer substrates (Figure 2c). The honeycomb pattern in the PMMA substrates served as the starting structure of the glancing angle deposition of Au films. By varying the deposition angle (50,60 and $\left.70^{\circ}\right)$, three different samples have been fabricated for each honeycomb interpore distance (Figure 2d-f).

In total, nine different samples have been prepared, each covering approximately $3 \mathrm{~cm}^{2}$, together with three reference samples of a deposition angle of $0^{\circ}$. Figure 3 a shows a photograph of the samples prepared at a deposition angle of $60^{\circ}$, the samples with $600 \mathrm{~nm}$ interpore distance show good diffraction (Figure $3 b$ ) while the samples with $430 \mathrm{~nm}$ interpore distance show moderate blue diffraction and the samples with $300 \mathrm{~nm}$ show no diffraction. However, the size of the samples are only limited by the size of the original molds, which were fabricated by another scalable technique, but the size of the samples was chosen to be compatible with commercial dismantled cuvettes and only a small area of the sample are probed at any given time. 


\section{NIL Process I}

\section{NIL Process II}

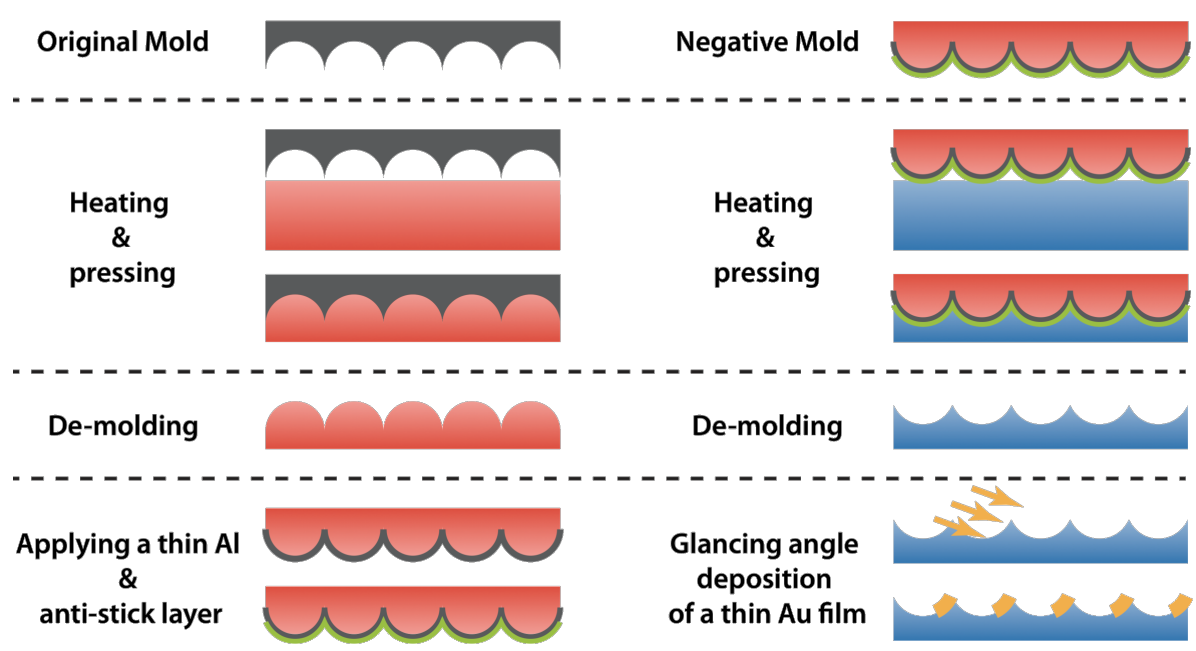

Figure 1: Schematical overview of the ECM fabrication process. First an original mold with a honeycomb structure is cast in a TOPAS polymer substrate. After de-molding a thin Al and anti-stick layer is applied to produce a negative mold. This negative mold is then cast in PMMA to produce the original honeycomb structure. This structure is used for initiation of glancing angle deposition of a $30 \mathrm{~nm}$ Au layer.

\section{The criteria for extrinsic chiral metamaterials}

A chiroptical response is only obtained from structures lacking mirror symmetry. As the unit structures of ECMs are not natively chiral, they rely greatly on the orientation of the sample with respect to the direction of the incident light causing a broken mirror symmetry. By careful design it is possible to design the ECM structure in such a way that no CD response is observed when the face of the ECM substrates are oriented perpendicular to the incident light. Hence a CD signal is only obtained when the sample is tilted out of one of the two angles $\theta$ and $\phi$ (Figure 4).

This enables the investigation of the enantiomeric ECM structures (right-handed and left-handed structure) to be probed in the same experiment, by inverting the tilt of the ECM with respect to the normal ECM face angle. The investigated ECMs have been designed in a way that it was possible to invert the CD signal by tilting the samples with respect to the $\theta$ angle while retaining a $\phi$ angle of $0^{\circ}$ (Figure 5). The observed response from the bare sample when irradiated with light at a normal incident angle may have three different causes: 1) various structural imperfections, 2) a spread in structure size, 3) the 3D nature of the ECM structure causing some intrinsic chirality. However, this $\mathrm{CD}$ response at $\phi=0^{\circ}$ has also been observed by ECMs produced with focused ion beam milling [13].

The ECM property that allows for the measurement of the enantiomeric structures from one sample, yields several advantages over PCMs in biosensor applications: 1) PCMs require fabrication of the two enantiomeric samples, which increases the cost and the risk of imperfections. 2) With the use of PCMs it is necessary to perform two independent experiments, which is time-consuming and difficult to implement into a commercial product. 3) Since the CD response is concentration-dependent, two independent PCM experiments are troublesome to interpret. All of these disadvantages with PCMs are totally avoided by the use of ECMs, which are both cheaper, more reliable and only require one experiment in biosensor applications.

\section{Probing the zero-response angle}

The zero-response angle of the ECM with $450 \mathrm{~nm}$ interpore distance and $50^{\circ}$ glancing angle deposition was identified by scanning the $\theta$ and $\phi$ angles separately (Figure 6). This ECM substrate was chosen as it exhibits more and stronger resonance modes than the other samples and will be used in most subsequent experiments. While the $\mathrm{CD}$ response was extremely sensitive towards angular rotation about the $\theta$ angle plane (Figure 6a), it exhibited a lower angular dependence on the $\phi$ angle plane (Figure 6b).

Furthermore, it is evident from the angular scans (Figure 6) that the ECM has an intrinsic left-hand chirality. Hence, the zeroresponse angle is not identified by the lowest $\mathrm{CD}$ response but as the center of the enantiomeric signals. From the $\theta$ angle scan (Figure 6a), it appears as if $\theta=-1^{\circ}$ and $\theta=+2^{\circ}$ display the least response. Hence, the $\theta$ angle resulting in minimum extrinsic chirality is between $0^{\circ}$ and $+1^{\circ}$. As virtually no difference in 

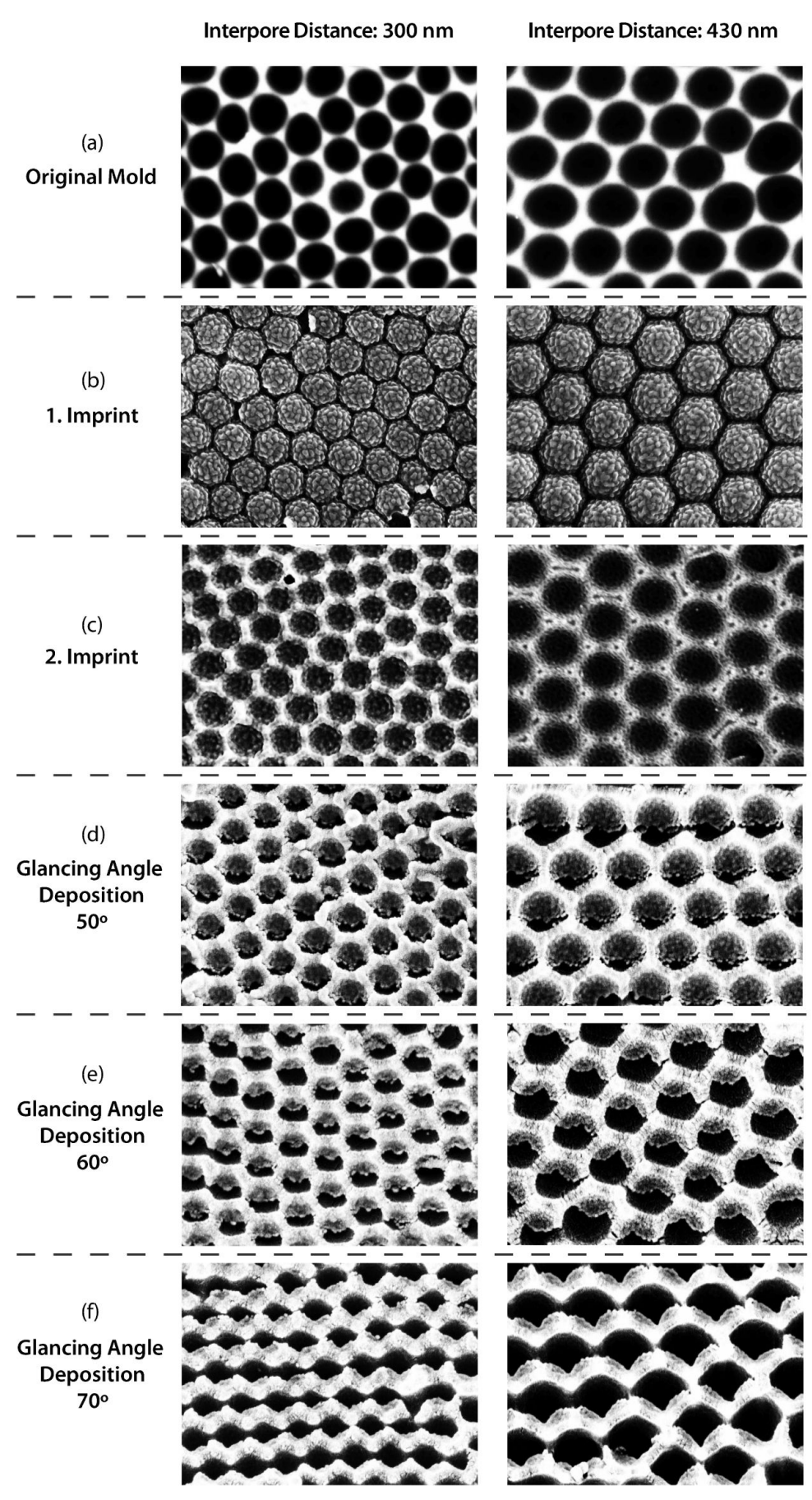

Interpore Distance: $600 \mathrm{~nm}$

(b)

1. Imprint

(1) 1.5.

10 0 .

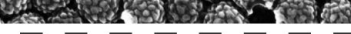

(1)

L $1-1=$

- 1 r. 1 r.

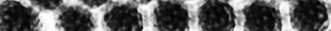

- trase
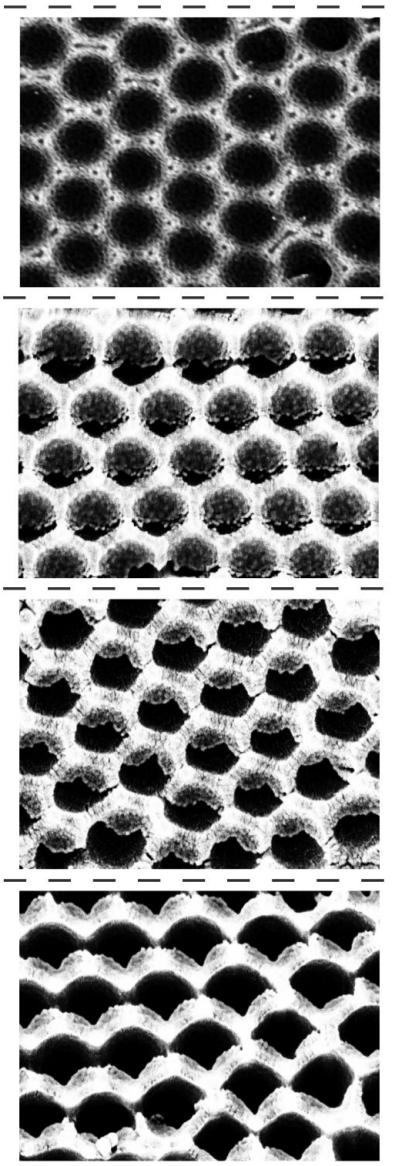

4060

6. 15
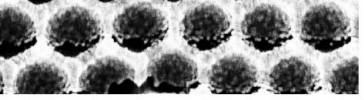
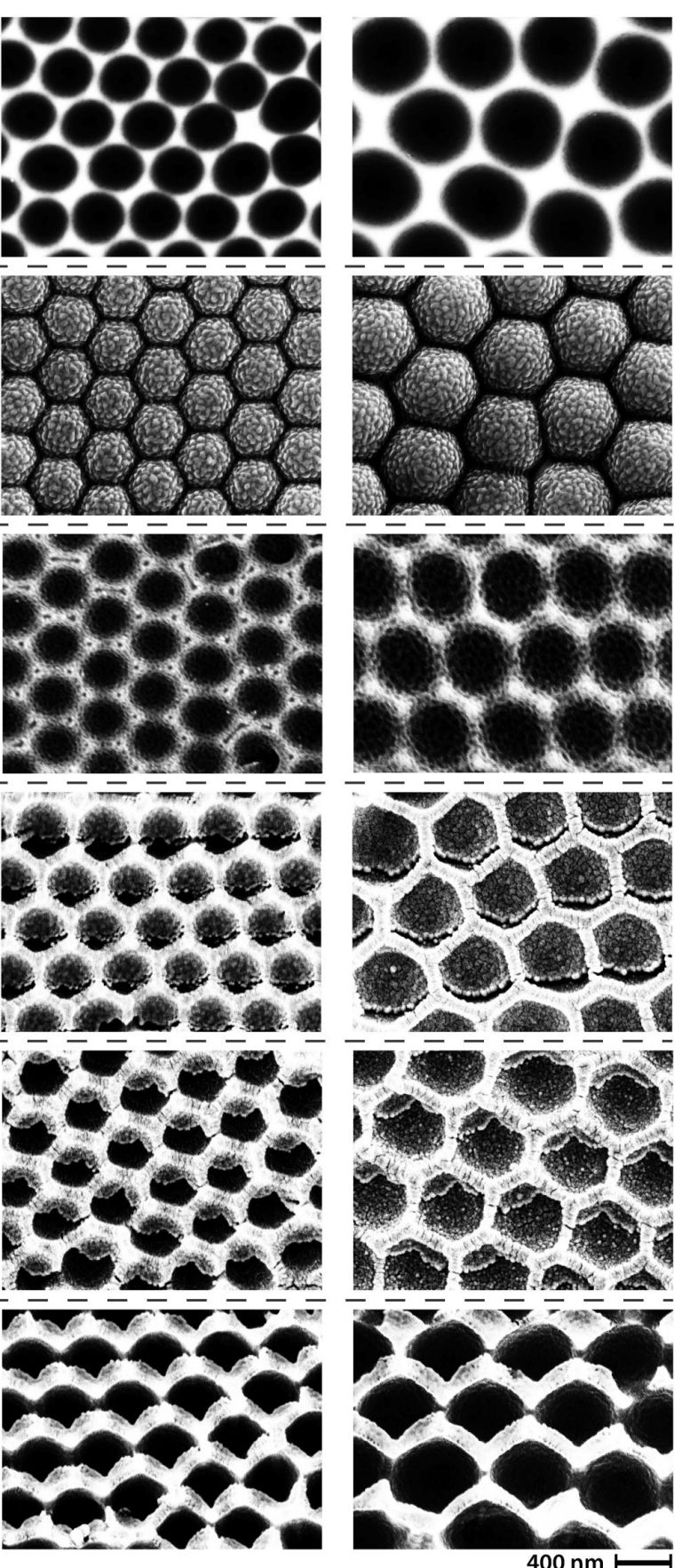

Figure 2: SEM pictures of (a) the original molds (b) 1. imprints with a thin Al layer (c) 2. imprints with a $2 \mathrm{~nm}$ Au layer and (d-f) all the different ECMs produced by glancing angle deposition of $30 \mathrm{~nm} A u$ at 50,60 and $70^{\circ}$. The scale bar is shown in the bottom right corner.

line shape is observed between $\theta=0^{\circ}$ and $\theta=+1^{\circ}, \theta=0^{\circ}$

was used as zero- $\theta$-angle throughout the paper for the sake of convenience.

The two resonance modes located in the wavelength range of $260-360 \mathrm{~nm}$ of the $\phi$ angle scan (Figure $6 \mathrm{~b}$ ) indicate that $\phi=0^{\circ}$ to $\phi=-3^{\circ}$ exhibits no extrinsic chirality. As virtually no difference in line shape is observed between these angles, $\phi=0^{\circ}$ was used as zero-response angle. Applying $\theta=0^{\circ}$ and $\phi=0^{\circ}$ as reference results in a more symmetrical angular scan (Figure 7) indicating the precision of the determination of the zeroresponse angle. 


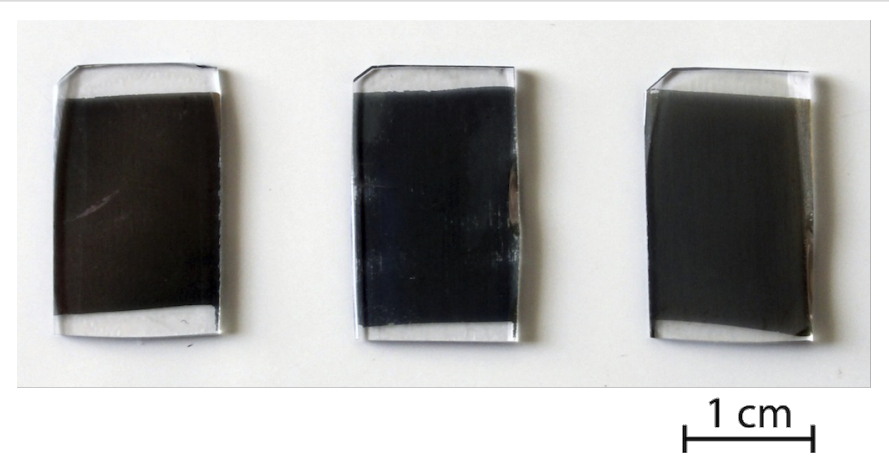

a)

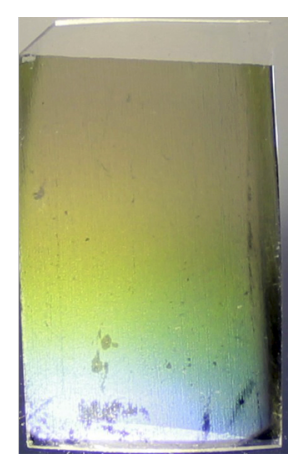

b)

Figure 3: (a) Photography of the ECM samples prepared at a deposition angle of $60^{\circ}$ and interpore distance of 300,430 and $600 \mathrm{~nm}$ from the left, respectively. The sizes were chosen as to be compatible with commercial dismantled cuvettes and only a small area of the sample is probed.

(b) Photography of the sample prepared by a deposition angle of $60^{\circ}$ and interpore distance of $600 \mathrm{~nm}$ with flashlight to illustrate the diffraction pattern (the scale bar does not apply to this photo).

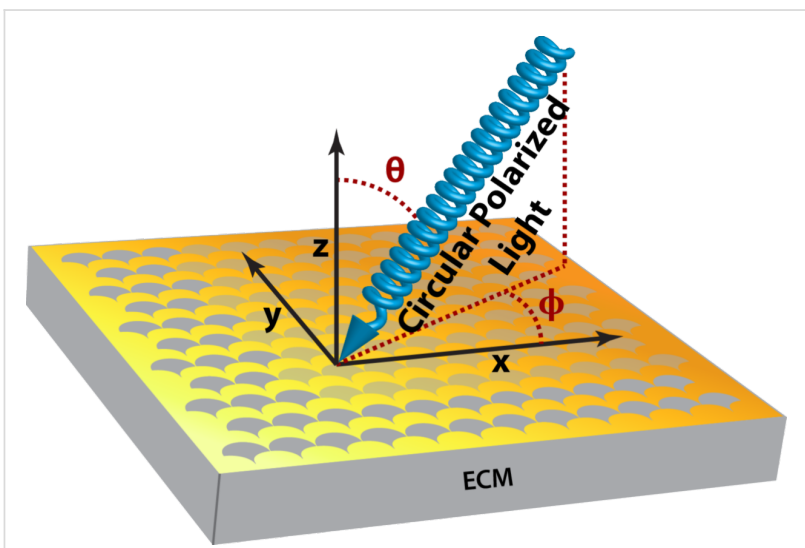

Figure 4: The two principle angles, $\theta$ and $\phi$, of incident light with respect to the ECM structures.

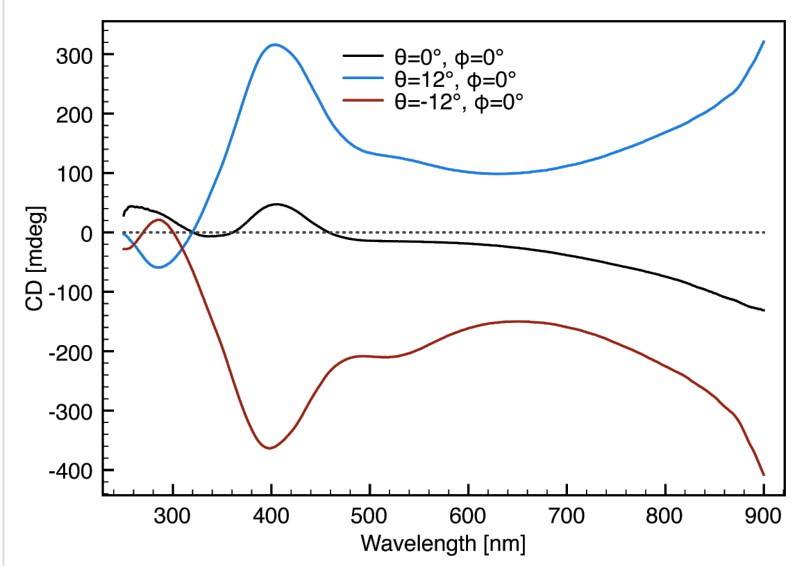

Figure 5: The resulting CD response from three ECM orientations $\left(\theta=0^{\circ}, \phi=0^{\circ}\right),\left(\theta=12^{\circ}, \phi=0^{\circ}\right)$ and $\left(\theta=-12^{\circ}, \phi=0^{\circ}\right)$ of the ECM with $300 \mathrm{~nm}$ interpore distance and $60^{\circ}$ deposition angle. It is evident that $\left(\theta=0^{\circ}, \phi=0^{\circ}\right)$ shows no significant response, while the two other orientations yield inverted line shapes.

\section{Influence of interpore distance and glancing angle deposition}

Structures with interpore distances of 300,430 and $600 \mathrm{~nm}$ exhibited main plasmonic resonance ranges of about 300-550 nm, 450-800 $\mathrm{nm}$ and above $900 \mathrm{~nm}$ (Figure 8).

Compared to the relatively narrow linewidth of the $\mathrm{CD}$ response from ECMs comprised of hole arrays, the honeycomb ECMs exhibit a rather broad signal [13]. This is presumably related to the heterogeneity and 3D nature of the ECM structures, which is less dominant in the hole arrays fabricated by focused ion beam lithography. In spite of this, the CD linewidths of the present ECMs are comparable to those of gammadion PCMs, which have been previously used for biosensor applications [10]. Furthermore, compared to PCMs fabricated by another scalable technique [29], the CD linewidths of the honeycomb ECMs are more narrow. Hence the heterogeneity and 3D structure has not limited the use of the honeycomb ECMs for biosensing applications.

While the interpore distance of the ECM array is the main factor in the position of the SPP resonance, the angle of the Au deposition has mainly an influence on the line shape and the number of resonance peaks (Figure 8). The samples prepared at 0 and $50^{\circ}$ glancing angle deposition show a similar line shape, as well as the samples prepared at 60 and $70^{\circ}$. This is reasonable as the angle deposition sensitivity, which determines the dependence of the resulting structure on the deposition angle, is very low at small angles but increases with large angles. The reason for this is found in the shadowing effect of the honeycomb structure. The glancing angle deposition is self-perpetuating at larger angles while small angles result only in minor shadowing effects. The samples prepared at 0 and $50^{\circ}$, in general, exhibit three to four distinct resonance peaks while the 


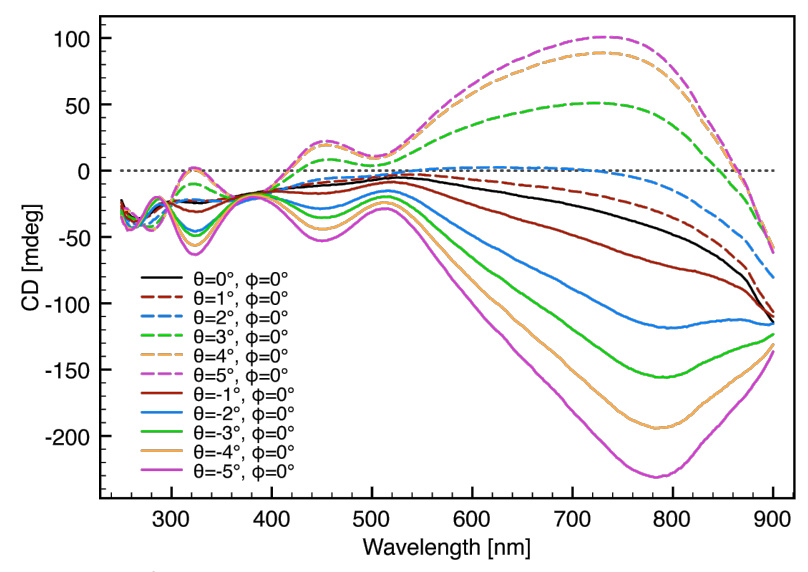

a)

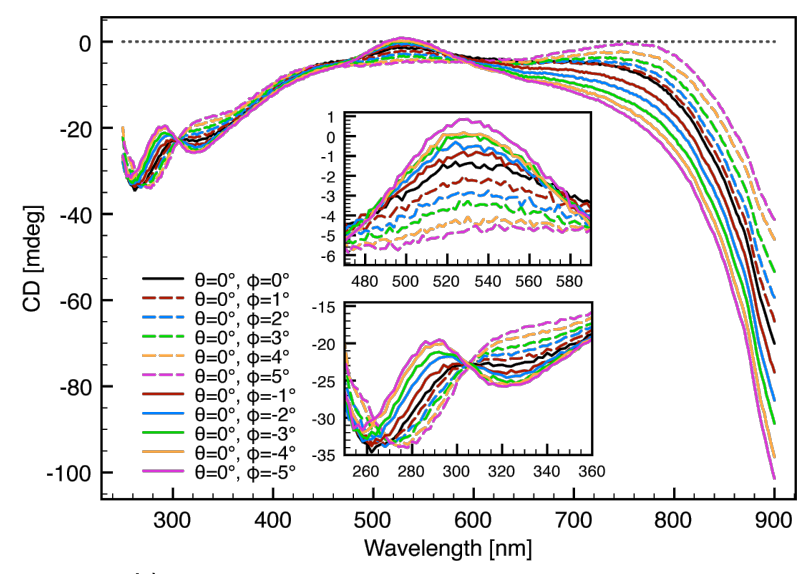

b)

Figure 6: Angular scans and corresponding CD response from the ECM fabricated with $430 \mathrm{~nm}$ interpore distance and $50^{\circ}$ glancing angle deposition (a) Independent scan of the $\theta$ angle defined in Figure 4. (b) Independent scan of the $\phi$ angle defined in Figure 4.

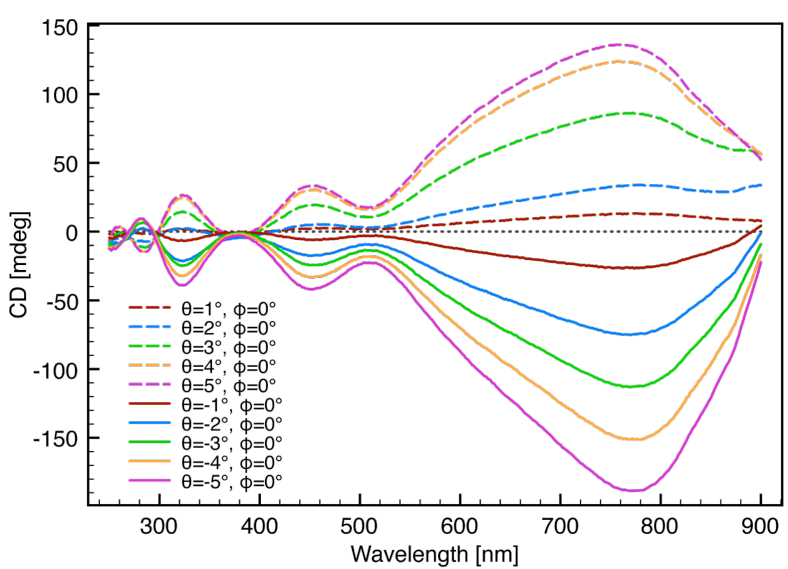

a)

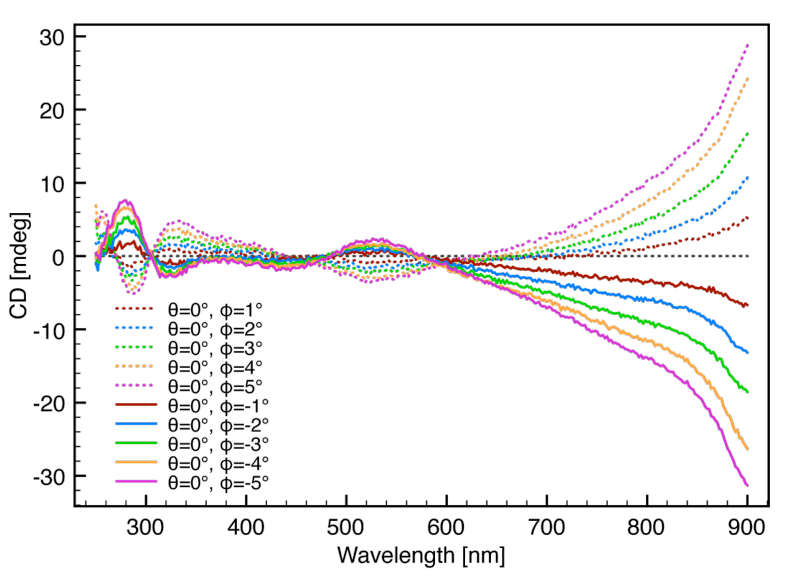

b)

Figure 7: Angular scans with the intrinsic chirality subtracted. A much higher symmetrical CD response from the ECM enantiomers is observed compared to the angular scans in Figure 6. (a) Independent scan of the $\theta$ angle. (b) Independent scan of the $\phi$ angle.

samples prepared at 60 and $70^{\circ}$ only exhibit two to three distinct resonance peaks. This might be explained by the gold inside the hole arrays produced at 0 and $50^{\circ}$ glancing angle deposition, resulting in a more complex 3D structure (Figure 2) and an additional origin of plasmonic resonance modes. The samples with $600 \mathrm{~nm}$ interpore distances had a main resonance wavelength above $900 \mathrm{~nm}$ (data not shown). These structures may find applications in other areas such as surface enhanced IR spectroscopy [41], terahertz generation [42] or THz-CD spectroscopy [43].

\section{Influence of the scanning angle and of intrinsic spatial structural variations}

As mentioned above, the plasmonic resonance wavelength and intensity depends on the orientation of the ECMs. Upon rotating the sample in the $\theta$ angle plane (Figure 9a) it is evident that the two distinct resonance modes in the near-UV region merge upon increasing the $\theta$ angle from 10 to $20^{\circ}$. At $\theta=30^{\circ}$ this peak is blue-shifted. When the $\theta$ angle is increased to 40 and $50^{\circ}$ the peak re-separates into two peaks. These changes may originate from the transition from a hole array towards a film with gratings upon increasing angles and subsequent larger backscattering. At $\theta=60^{\circ}$ the backscattering has increased significantly, resulting in a pronounced drop in CD response. Furthermore, the $770 \mathrm{~nm}$ plasmonic resonance mode does not show any angular dependence and remains located at the same wavelength.

From the $\phi$ angle scan (Figure $9 b$ ) it is evident that only plasmonic resonance modes in the infrared and UV range are in- 

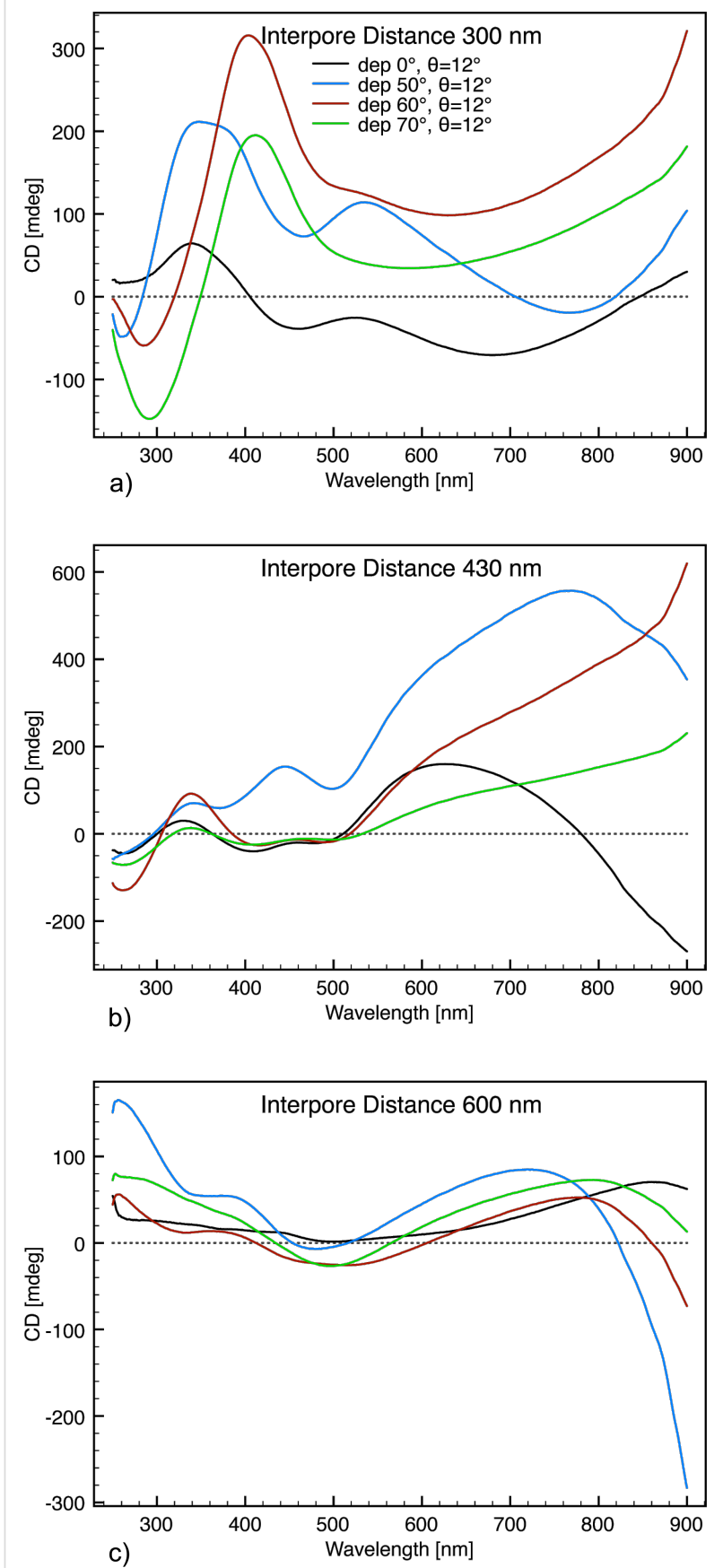

Figure 8: $C D$ response from all the produced ECMs. (a) The CD response from the structures with $300 \mathrm{~nm}$ interpore distances. (b) The response from the structures with $430 \mathrm{~nm}$ interpore distances. (c) The response from the structures with $600 \mathrm{~nm}$ interpore distances. All measurements were recorded with an ECM orientation of $\theta=12^{\circ}$ and $\phi=0^{\circ}$.

duced. Upon increasing the $\phi$ angle, the resonance modes in the UV range intensifies and changes of the resonance wavelengths occur. It is also noteworthy for other applications of the ECM, that a very strong resonance mode in the range of 900-1100 nm has been identified in other measurements (data not shown) but is outside of the measured range presented here as it is not relevant to protein sensing using CD spectroscopy. Similar to the $\theta$ angular scan, the $\mathrm{CD}$ response at a $\phi=60^{\circ}$ drops as a consequence of significantly increased backscattering.

Typically, metamaterials are fabricated with a significantly smaller surface area than the beam profile used to probe the sample. However, the present ECMs are significantly larger, but with various structural imperfections. To validate the integrity of the sample fabricated with $430 \mathrm{~nm}$ interpore distance and a glancing angle deposition of $50^{\circ}$ was probed in two different positions over $6 \mathrm{~mm}$ apart. Minor differences were observed (Figure 10), indicating small structural differences.

These differences are no larger than what has been reported for PCMs fabricated with e-beam lithography [10] or ECMs fabricated with ion beam milling [13] and most likely originate from structural imperfections. Another contributing factor might be that the ECM surface is much larger than the beam profile, hence the structures in the circumference of the beam profile may appear different from those substructures being completely probed. Beam profile induced imperfections are circumvented in usual fabrication techniques as the achievable sample area is much smaller than the beam profile.

\section{Sensing of chiral molecules}

As a proof of concept, the sensing power of the honeycomb ECMs were investigated by monitoring the resonance shift upon adsorption of the chiral organic molecule cysteamine and the protein cytochrome c. cytochrome c acts as an electron shuttle and as a respiratory redox protein [44]. It also assists as an important mediator in the apoptotic pathways [44]. Due to a free surface-accessible cysteine it is well known to readily adsorb onto gold surfaces and is often used to study electron transfer in cyclic voltammetry [45]. Cysteamine is the simplest stable aminothiol, hence it readily adsorbs onto gold surfaces and forms a self-assembled monolayer which is often used as the first layer in the functionalization of surfaces [46].

In all experiments the ECM fabricated with $430 \mathrm{~nm}$ interpore distance and a glancing deposition angle of $50^{\circ}$ was used as it displays a well-defined response at ca. $770 \mathrm{~nm}$ compared to the other structures, together with two minor distinct peaks at ca. $440 \mathrm{~nm}$ and ca. $330 \mathrm{~nm}$. The resulting spectra are presented in Figure 11 and the corresponding shifts compared to the bare structure are summarized in Table 1.

A similar plasmonic resonance shift of the resonance mode around $770 \mathrm{~nm}$ upon adsorption of cytochrome c was observed at different $\theta$ angles (Figure 12 and Table 2). This indicates that the sensitivity of the plasmonic resonance towards changes in 

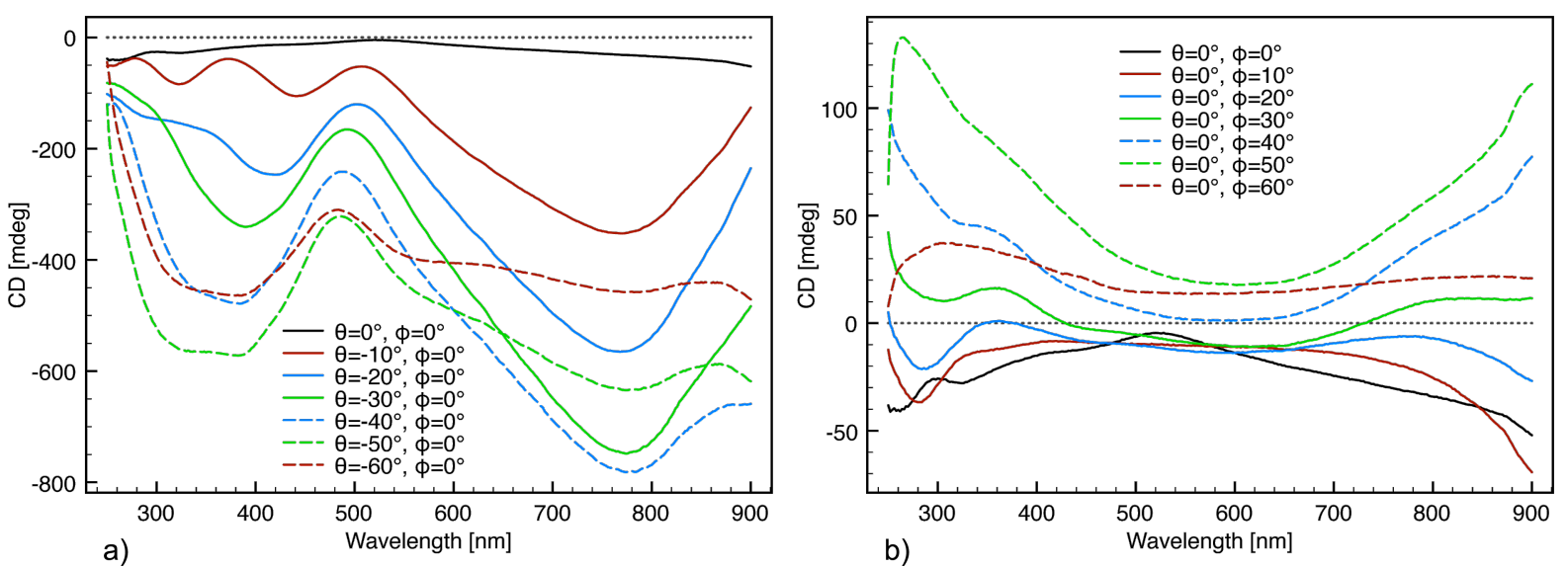

Figure 9: Angular scans with large angle increments and corresponding the CD response from the ECM fabricated with $430 \mathrm{~nm}$ interpore distance and $50^{\circ}$ glancing angle deposition. (a) Independent scan of the $\theta$ angle. (b) Independent scan of the $\phi$ angle.

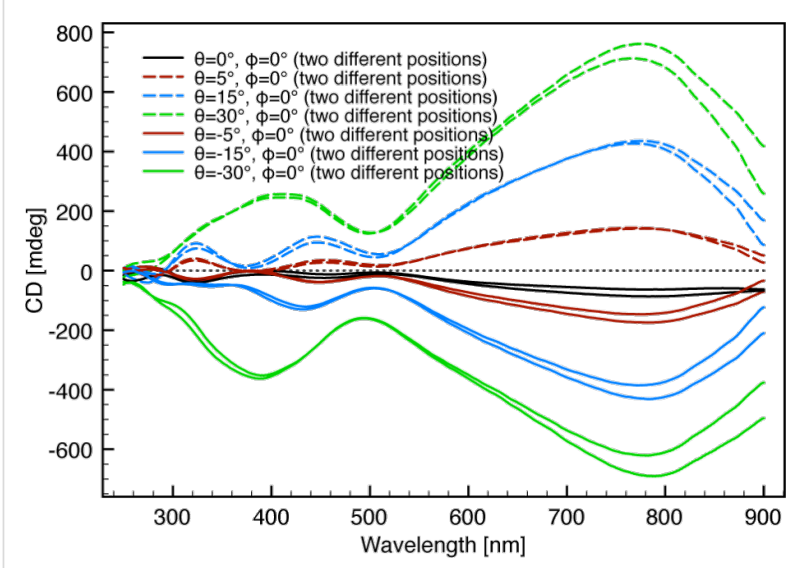

Figure 10: $C D$ response from the ECM fabricated with $430 \mathrm{~nm}$ interpore distance and $50^{\circ}$ glancing angle deposition in four different angle orientation and measured in two different positions $6 \mathrm{~mm}$ apart on the sample.

the local refractive index near the ECM surface is not effected by the $\theta$ angle. Only the resonance mode around $770 \mathrm{~nm}$ was used in this analysis as the resonances of the other modes are altered as described above and as a result are incomparable.

It has been demonstrated that the dissymmetry factor [10] $(\Delta \Delta \lambda)$ of the shifts of the right-handed spectrum compared to the left-handed spectrum $\left(\Delta \Delta \lambda=\Delta \lambda_{\text {right }}-\Delta \lambda_{\text {left }}\right)$ is an indication of an anisotropic adsorption (Table 3) [10]. Whereas a perfect isotropic adsorption would result in a $\Delta \Delta \lambda$ value of zero. Interestingly, the adsorption of the two molecules does not result in the same behavior in signal shifts. While the adsorption of cytochrome c did not result in a measurable dissymmetry shift, the adsorption of cysteamine caused a relatively large dissymmetry shift as indicated by the $770 \mathrm{~nm}$ signal. This
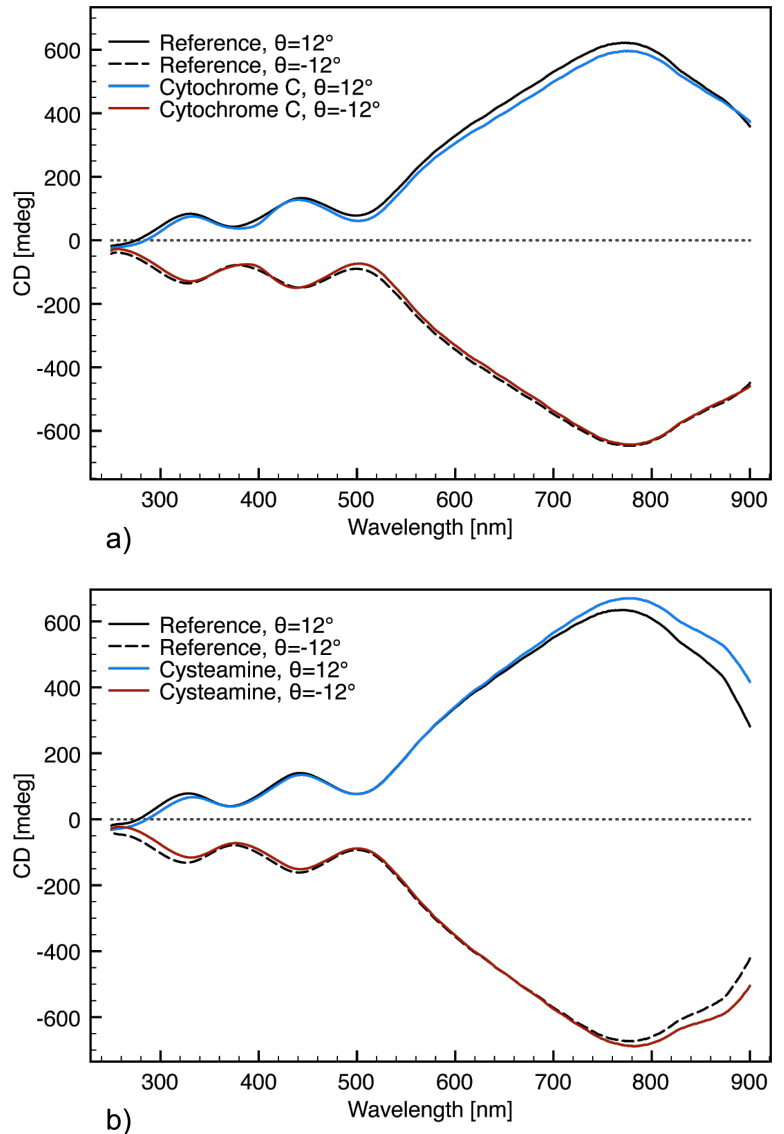

Figure 11: CD response from a bare ECM and with a molecule adsorbed on the surface. (a) $C D$ response with and without cytochrome $c$ adsorbed on the ECM surface. (b) CD response with and without Cysteamine adsorbed on the ECM surface. All measurements were recorded with an ECM orientation of $\theta=12^{\circ} ; \phi=0^{\circ}$ or $\theta=-12^{\circ}$; $\phi=0^{\circ}$. 
Table 1: Excitation shifts originating from different molecules.

\begin{tabular}{lrrr} 
molecule & $\begin{array}{r}\Delta \lambda_{770 \mathrm{~nm}} \\
\left(\theta_{12} / \theta_{-12}\right)\end{array}$ & $\begin{array}{r}\Delta \lambda_{440 \mathrm{~nm}} \\
\left(\theta_{12} / \theta_{-12}\right)\end{array}$ & $\begin{array}{r}\Delta \lambda_{330} \mathrm{~nm} \\
\left(\theta_{12} / \theta_{-12}\right)\end{array}$ \\
\hline cytochrome c & $2 / 2 \mathrm{~nm}$ & $-4 /-4 \mathrm{~nm}$ & $2 / 3 \mathrm{~nm}$ \\
cysteamine & $7 / 12 \mathrm{~nm}$ & $2 / 2 \mathrm{~nm}$ & $4 / 5 \mathrm{~nm}$
\end{tabular}

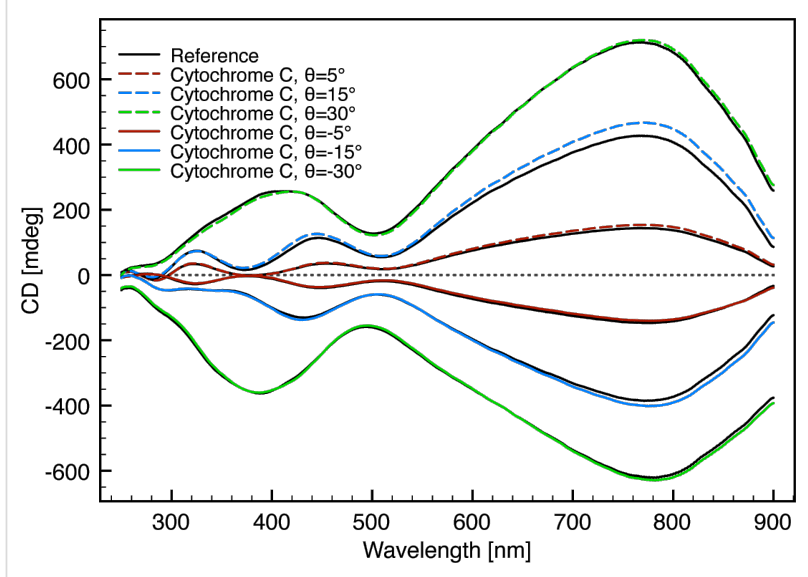

Figure 12: CD response from the ECM fabricated with $430 \mathrm{~nm}$ interpore distance and $50^{\circ}$ glancing angle deposition with different angle orientation and measured with and without cytochrome $c$ adsorbed on the surface.

Table 2: Excitation shifts and dissymmetry factors of the plasmonic resonance at $770 \mathrm{~nm}$ at different $\theta$ angles, originating from adsorption of cytochrome $c$ on the ECM fabricated with $430 \mathrm{~nm}$ interpore distance and a glancing angle deposition of $50^{\circ}$.

\begin{tabular}{lll} 
angle & $\Delta \lambda_{770 \mathrm{~nm}}$ & $\Delta \Delta \lambda_{770 \mathrm{~nm}}$ \\
\hline$\left(\theta_{5} / \theta_{-5}\right)$ & $1.4 / 1.5 \mathrm{~nm}$ & $0.1 \mathrm{~nm}$ \\
$\left(\theta_{15} / \theta_{-15}\right)$ & $2.3 / 2.6 \mathrm{~nm}$ & $0.3 \mathrm{~nm}$ \\
$\left(\theta_{30} / \theta_{-30}\right)$ & $2.2 / 2.4 \mathrm{~nm}$ & $0.2 \mathrm{~nm}$
\end{tabular}

Table 3: Dissymmetry factors, calculated from the shifts given in Table 1.

\begin{tabular}{lrrr} 
molecule & $\Delta \Delta \lambda_{770 \mathrm{~nm}}$ & $\Delta \Delta \Lambda_{440 \mathrm{~nm}}$ & $\Delta \Delta \Lambda_{330 \mathrm{~nm}}$ \\
\hline cytochrome c & $0 \mathrm{~nm}$ & $0 \mathrm{~nm}$ & $-1 \mathrm{~nm}$ \\
cysteamine & $-5 \mathrm{~nm}$ & $0 \mathrm{~nm}$ & $-1 \mathrm{~nm}$
\end{tabular}

is coherent with other studies, demonstrating that cysteamine forms a well-ordered layer on Au surfaces [47,48]. Furthermore, it has been reported that cytochrome $\mathrm{c}$ either adsorbs with the $\alpha$-helix structure parallel to the Au surface [49] or that the protein instantly denaturates upon adsorption to the Au surface [50]. In either case this will result in an isotropic layer with respect to the incoming light, which is coherent with the obtained results.

The average extent of the two plasmonic enantiomer shifts $\left(\Delta \lambda_{\text {av }}=\left(\Delta \lambda_{\text {right }}+\Delta \lambda_{\text {left }}\right) / 2\right.$ gives an indication of the amount of molecules adsorbed on the gold surface of the metamaterial [10]. Instead of using the peak at $550 \mathrm{~nm}$ to determine the surface coverage as previously reported by PCMs [10], the peak at $330 \mathrm{~nm}$ has been used in this paper, since it is the peak located closest to the UV range (Table 4).

Table 4: Average shifts calculated from the shifts given in Table 1.

\begin{tabular}{ll} 
molecule & $\Delta \lambda_{\mathrm{AV}, 330 \mathrm{~nm}}$ \\
\hline cytochrome c & $2.5 \mathrm{~nm}$ \\
cysteamine & $4.5 \mathrm{~nm}$
\end{tabular}

From the data it is apparent that both cytochrome c and cysteamine do interact with the ECM gold surface. Compared to another study using PCMs, it is apparent that the $\Delta \lambda_{\mathrm{AV}, 330 \mathrm{~nm}}$ of cytochrome $\mathrm{c}$ and cysteamine is comparable to the $\Delta \lambda_{\mathrm{AV}, \approx 550 \mathrm{~nm}}$ of the proteins resulting from the highest adsorption on a PCMs gold surface [10]. As the sensitivity of the ECM resonance mode at $330 \mathrm{~nm}$ is unknown the exact surface coverage cannot be determined. However, the sensitivity is not expected to deviate significantly from that observed with PCMs at $550 \mathrm{~nm}$. Based on this both cytochrome $\mathrm{c}$ and cysteamine are adsorbed in picogram quantities on the ECM Au surface.

The current results demonstrate that proteins and chiral organic molecules in general readily adsorb onto the ECM surface. This demonstrates that the ECMs may be used for detection of proteins and chiral organic molecules in a label-free way. Furthermore, the ease of cysteamine adsorption on the ECM surface suggests that other molecules such as ethanedithiol may be used to functionalize the ECM surface and provide the possibility to fabricate very sensitive sensor arrays.

\section{Conclusion}

In conclusion, a strong chiroptical response has been demonstrated from different achiral plasmonic hole arrays. The arrays were fabricated by a scaleable technique while retaining control and order of the resulting arrays. This signifies a substantial improvement to standard fabrication methods such as focused ion beam and electron beam lithography concerning cost and production time. Furthermore, the use of a small chiral organic molecule and a protein has been used as a proof of concept for the sensing of biological and chiral organic molecules in picogram quantities by $\mathrm{CD}$ spectroscopy. The response of the presented ECMs was tunable within the UVA and IR regions, 
depending mainly on the interpore distance of the hole arrays. The deposition angle had an effect on the amount of resonance modes. Small glancing angle deposition resulted in complex ECM geometries and consequently more resonance modes were observed. The number of modes is larger than that which has been previously reported for PCMs [10,32,34]. This is a significant improvement, as more signals yield a more detailed protein fingerprint.

The enantiomeric form of the investigated ECMs were recorded by inverting the tilt of the same sample about the $\theta$ angle plane, effectively eliminating the disadvantages of PCMs having to fabricate two independent samples and conducting two separate experiments to probe the protein fingerprint. CD spectroscopy revealed that both cytochrome $\mathrm{c}$ and cysteamine readily adsorbed on the ECM gold surface, amounting to picogram quantities. Furthermore, the formation of a cysteamine layer on the ECM gold surface suggests that similar organic molecules may be used to fabricate functionalized surfaces applicable for sensors with increased sensitivity or arrays hereof in a cheap and scaleable way.

\section{Experimental}

\section{Fabrication of extrinsic chiral metamaterials}

The original molds were prepared by anodic aluminum oxidation using a custom-built anodization and wet-etching system. Al foils (99.98\%, Advent Research Materials Ltd. AL103310) were used as substrates after cleaning in an ultrasonic bath with a sequence of acetone, deionized water and methanol for $1 \mathrm{~min}$ each. In total three types of molds were prepared with different interpore distances: 300, 430 and $600 \mathrm{~nm}$. The substrate with $300 \mathrm{~nm}$ interpore distance was prepared by anodization in $0.3 \mathrm{M}$ oxalic acid solution at $140 \mathrm{~V}$ and with a solution temperature of $283 \pm 0.5 \mathrm{~K}$ for $40 \mathrm{~min}$. The substrate with $430 \mathrm{~nm}$ interpore distance was prepared by anodization in $1 \mathrm{M}$ phosphoric acid solution at $180 \mathrm{~V}$ and with a solution temperature of $273 \pm 0.5 \mathrm{~K}$ for $100 \mathrm{~min}$. The substrate with $600 \mathrm{~nm}$ interpore distance was prepared by anodization in $2 \mathrm{M}$ citric acid solution at $285 \mathrm{~V}$ and with a solution temperature of $293 \pm 0.5 \mathrm{~K}$ for $20 \mathrm{~min}$. More details on the fabrication of the original mold has been reported previously [39]. The original molds were used to make negative imprints by thermal nanoimprint lithography using the EVG520HE semi-automated hot embossing system. This was done in TOPAS 5013L-10 substrates under vacuum with a stamping pressure of 1.25 bar and at $160^{\circ} \mathrm{C}$ using the original molds. Next a $30 \mathrm{~nm}$ film was sputter-coated on the surface of the negative imprints. A monolayer of trichloro$(1 H, 1 H, 2 H, 2 H$-perfluorooctyl)silane ( $25 \mathrm{vol} \%$ in toluene) was applied to the Al films by gas phase deposition for $1 \mathrm{~h}$ in a vacuum desiccator. These samples served as new molds for the second imprint in PMMA. The parameters of this imprint were similar to the once described above but were prepared at $120^{\circ} \mathrm{C}$. The negative molds were used several times without observable deterioration. The final ECMs were achieved by glancing angle deposition of $2 \mathrm{~nm} \mathrm{Cr}$ followed by $50 \mathrm{~nm} \mathrm{Au}$ at different deposition angles.

\section{Scanning electron microscopy}

SEM measurements of the ECM surfaces were done in high vacuum $\left(1 \cdot 10^{-6}\right.$ mbar $)$ and an accelerating voltage $10 \mathrm{kV}$ using a Zeiss $1540 \mathrm{XB}$ system and standard procedure. The samples were coated by $2 \mathrm{~nm}$ gold as to prevent a buildup of static charge.

\section{CD spectroscopy measurements}

The ECMs were compatible with commercial available liquid CD cells with a path length of $0.1 \mathrm{~mm}$ and a total volume of $50 \mu \mathrm{L}$. The CD spectra were collected in normal incident mode where the samples were parallel to the detector and in tilted configuration, where the samples were tilted by $12^{\circ}$. CD spectra were collected using a commercial JASCO J-750 spectropolarimeter.

\section{Adsorption of cytochrome $\mathrm{c}$ and cysteamine onto extrinsic chiral materials}

Cytochrome $\mathrm{c}$ was adsorbed on the substrates by incubation of $50 \mu \mathrm{L}$ protein solution $\left(1 \mathrm{mg} \cdot \mathrm{mL}^{-1}\right)$ for $1 \mathrm{~h}$. Cysteamine was adsorbed by incubating $50 \mu \mathrm{L}$ of solution $(10 \mathrm{mM})$ for $24 \mathrm{~h}$. The cysteamine solution was prepared with degassed Milli-Q water and the cytochrome c solution was prepared using $5 \mathrm{mM}$ PBS buffer at $\mathrm{pH} 7.4$.

\section{Surface regeneration of extrinsic chiral materials}

ECMs were reused several times and before each experiment the substrates were submerged for $2 \mathrm{~h}$ in a sodium dodecyl sulfate solution, followed by a 30 min bath in a Hellmanex III solution at $37{ }^{\circ} \mathrm{C}$. Finally, the substrates were cleaned in an oxygen-plasma cleaning unit for $1 \mathrm{~h}$. After each step the ECMs were rinsed with Milli-Q water.

\section{References}

1. Valentine, J.; Zhang, S.; Zentgraf, T.; Ulin-Avila, E.; Genov, D. A.; Bartal, G.; Zhang, X. Nature 2008, 455, 376-379. doi:10.1038/nature07247

2. Atwater, H. A.; Polman, A. Nat. Mater. 2010, 9, 205-213. doi:10.1038/nmat2629

3. Zia, R.; Schuller, J. A.; Chandran, A.; Brongersma, M. L. Mater. Today 2006, 9, 20-27. doi:10.1016/S1369-7021(06)71572-3

4. Abb, M.; Wang, Y.; Papasimakis, N.; de Groot, C. H.; Muskens, O. L. Nano Lett. 2014, 14, 346-352. doi:10.1021/nl404115g

5. Kühler, P.; Weber, M.; Lohmüller, T. ACS Appl. Mater. Interfaces 2014, 6, 8947-8952. doi:10.1021/am5023418 
6. Yanik, A. A.; Huang, M.; Kamohara, O.; Artar, A.; Geisbert, T. W.; Connor, J. H.; Altug, H. Nano Lett. 2010, 10, 4962-4969. doi:10.1021/nl103025u

7. Pendry, J. B. Science 2004, 306, 1353-1355. doi:10.1126/science.1104467

8. Zhang, S.; Park, Y.-S.; Li, J.; Lu, X.; Zhang, W.; Zhang, X. Phys. Rev. Lett. 2009, 102, 023901. doi:10.1103/PhysRevLett.102.023901

9. Hannam, K.; Powell, D. A.; Shadrivov, I. V.; Kivshar, Y. S. Phys. Rev. B 2014, 89, 125105. doi:10.1103/PhysRevB.89.125105

10. Hendry, E.; Carpy, T.; Johnston, J.; Popland, M.; Mikhaylovskiy, R. V.; Lapthorn, A. J.; Kelly, S. M.; Barron, L. D.; Gadegaard, N.; Kadodwala, M. Nat. Nanotechnol. 2010, 5, 783-787. doi:10.1038/nnano.2010.209

11. Bunn, C., Ed. Chemical Crystallography; Oxford University Press: Oxford, United Kingdom, 1945.

12. Plum, E.; Liu, X.-X.; Fedotov, V. A.; Chen, Y.; Tsai, D. P.; Zheludev, N. I. Phys. Rev. Lett. 2009, 102, 113902. doi:10.1103/PhysRevLett.102.113902

13. Maoz, B. M.; Ben Moshe, A.; Vestler, D.; Bar-Elli, O.; Markovich, G Nano Lett. 2012, 12, 2357-2361. doi:10.1021/nl300316f

14. Zu, S.; Bao, Y.; Fang, Z. Nanoscale 2016, 8, 3900-3905. doi:10.1039/C5NR09302C

15. Zambrana-Puyalto, X.; Vidal, X.; Molina-Terriza, G. Nat. Commun. 2014, 5, 4922. doi:10.1038/ncomms5922

16. Arteaga, O.; Maoz, B. M.; Nichols, S.; Markovich, G.; Kahr, B. Opt. Express 2014, 22, 13719-13732. doi:10.1364/OE.22.013719

17. Feng, C.; Wang, Z. B.; Lee, S.; Jiao, J.; Li, L. Opt. Commun. 2012, 285 2750-2754. doi:10.1016/j.optcom.2012.01.062

18. Shi, J. H.; Zhu, Z.; Ma, H. F.; Jiang, W. X.; Cui, T. J. J. Appl. Phys. 2012, 112, 073522. doi:10.1063/1.4757961

19. Wang, F.; Wang, Z.; Shi, J. J. Appl. Phys. 2014, 116, 153506. doi:10.1063/1.4898314

20. Sersic, I.; van de Haar, M. A.; Arango, F. B.; Koenderink, A. F. Phys. Rev. Lett. 2012, 108, 223903. doi:10.1103/PhysRevLett.108.223903

21. Yannopapas, V. Opt. Lett. 2009, 34, 632-634. doi:10.1364/OL.34.000632

22. Shaltout, A.; Liu, J.; Shalaev, V. M.; Kildishev, A. V. Nano Lett. 2014, 14, 4426-4431. doi:10.1021/nl501396d

23. Hashiyada, S.; Narushima, T.; Okamoto, H. J. Phys. Chem. C 2014, 118, 22229-22233. doi:10.1021/jp507168a

24. Kruk, S. S.; Helgert, C.; Decker, M.; Staude, I.; Menzel, C.; Etrich, C.; Rockstuhl, C.; Jagadish, C.; Pertsch, T.; Neshev, D. N.; Kivshar, Y. S. Phys. Rev. B 2013, 88, 201404-201409. doi:10.1103/PhysRevB.88.201404

25. Boutria, M.; Oussaid, R.; Van Labeke, D.; Baida, F. I. Phys. Rev. B 2012, 86, 155428-155435. doi:10.1103/PhysRevB.86.155428

26. Belardini, A.; Benedetti, A.; Centini, M.; Leahu, G.; Mura, F.; Sennato, S.; Sibilia, C.; Robbiano, V.; Giordano, M. C.; Martella, C.; Comoretto, D.; de Mongeot, F. B. Adv. Opt. Mater. 2014, 2, 208-213. doi:10.1002/adom.201300385

27. Yokoyama, A.; Yoshida, M.; Ishii, A.; Kato, Y. K. Phys. Rev. X 2014, 4, 11005-11011. doi:10.1103/PhysRevX.4.011005

28. Singh, J. H.; Nair, G.; Ghosh, A.; Ghosh, A. Nanoscale 2013, 5 , 7224-7228. doi:10.1039/C3NR02666C

29. Yeom, B.; Zhang, H.; Zhang, H.; Park, J. I.; Kim, K.; Govorov, A. O.; Kotov, N. A. Nano Lett. 2013, 13, 5277-5283. doi:10.1021/nl402782d
30. Schreiber, R.; Do, J.; Roller, E.-M.; Zhang, T.; Schüller, V. J.; Nickels, P. C.; Feldmann, J.; Liedl, T. Nat. Nanotechnol. 2014, 9 , 74-78. doi:10.1038/nnano.2013.253

31. Zhou, Y.; Yang, M.; Sun, K.; Tang, Z.; Kotov, N. A. J. Am. Chem. Soc. 2010, 132, 6006-6013. doi:10.1021/ja906894r

32. Chen, W.; Bian, A.; Agarwal, A.; Liu, L.; Shen, H.; Wang, L.; Xu, C.; Kotov, N. A. Nano Lett. 2009, 9, 2153-2159. doi:10.1021/n1900726s

33. Shen, X.; Asenjo-Garcia, A.; Liu, Q.; Jiang, Q.; García de Abajo, F. J.; Liu, N.; Ding, B. Nano Lett. 2013, 13, 2128-2133. doi:10.1021/nl400538y

34. Fan, Z.; Govorov, A. O. Nano Lett. 2010, 10, 2580-2587. doi:10.1021/nl101231b

35. Nemiroski, A.; Gonidec, M.; Fox, J. M.; Jean-Remy, P.; Turnage, E.; Whitesides, G. M. ACS Nano 2014, 8, 11061-11070. doi:10.1021/nn504214b

36. Chang, Y.-C.; Lu, S.-C.; Chung, H.-C.; Wang, S.-M.; Tsai, T.-D.; Guo, T.-F. Sci. Rep. 2013, 3, 3339. doi:10.1038/srep03339

37. Cataldo, S.; Zhao, J.; Neubrech, F.; Frank, B.; Zhang, C.; Braun, P. V.; Giessen, H. ACS Nano 2012, 6, 979-985. doi:10.1021/nn2047982

38. Oates, T. W. H.; Shaykhutdinov, T.; Wagner, T.; Furchner, A.; Hinrichs, K. Adv. Mater. 2014, 26, 7197-7201. doi:10.1002/adma.201402012

39. Tsao, Y.-C.; Søndergaard, T.; Skovsen, E.; Gurevich, L.; Pedersen, K.; Pedersen, T. G. Opt. Express 2013, 21, A84-A95. doi:10.1364/OE.21.000A84

40. Tsao, Y.-C.; Fisker, C.; Pedersen, T. G. Opt. Express 2014, 22, A651-A662. doi:10.1364/OE.22.00A651

41. Li, Y.; Su, L.; Shou, C.; Yu, C.; Deng, J.; Fang, Y. Sci. Rep. 2013, 3, 2865. doi:10.1038/srep02865

42. Luo, L.; Chatzakis, I.; Wang, J.; Niesler, F. B. P.; Wegener, M.; Koschny, T.; Soukoulis, C. M. Nat. Commun. 2014, 5, 3055. doi:10.1038/ncomms4055

43. Choi, J.-H.; Cho, M. J. Phys. Chem. B 2014, 118, 12837-12843. doi:10.1021/jp508547y

44. Liu, X.; Kim, C. N.; Yang, J.; Jemmerson, R.; Wang, X. Cell 1996, 86 147-157. doi:10.1016/S0092-8674(00)80085-9

45. Liu, H.; Yamamoto, H.; Wei, J.; Waldeck, D. H. Langmuir 2003, 19, 2378-2387. doi:10.1021/la026378n

46. Shervedani, R. K.; Farahbakhsh, A.; Bagherzadeh, M. Anal. Chim. Acta 2007, 587, 254-262. doi:10.1016/j.aca.2007.01.053

47. Wirde, M.; Gelius, U.; Nyholm, L. Langmuir 1999, 15, 6370-6378. doi:10.1021/la9903245

48. Shervedani, R. K.; Bagherzadeh, M.; Mozaffari, S. A. Sens. Actuators, B 2006, 115, 614-621. doi:10.1016/j.snb.2005.10.027

49. Lin, S.; Jiang, X.; Wang, L.; Li, G.; Guo, L. J. Phys. Chem. C 2012, 116, 637-642. doi:10.1021/jp2063782

50. Zhou, Y.; Nagaoka, T.; Zhu, G. Biophys. Chem. 1999, 79, 55-62. doi:10.1016/S0301-4622(99)00038-1 


\section{License and Terms}

This is an Open Access article under the terms of the Creative Commons Attribution License

(http://creativecommons.org/licenses/by/2.0), which permits unrestricted use, distribution, and reproduction in any medium, provided the original work is properly cited.

The license is subject to the Beilstein Journal of Nanotechnology terms and conditions:

(http://www.beilstein-journals.org/bjnano)

The definitive version of this article is the electronic one which can be found at:

doi:10.3762/bjnano.7.83 